Research Article

\title{
Grey Relation Evaluation and Truth Value Fusion of Rolling Bearing Multisource Residual Stress
}

\author{
Xiaoxu Pang $\mathbb{D}^{1,2,3}$ Dongfeng Wang, ${ }^{4}$ Lihai Chen, ${ }^{1,3}$ and Liang Ye ${ }^{5}$ \\ ${ }^{1}$ School of Mechanical and Electrical Engineering, Henan University of Science and Technology, Luoyang, Henan 471003, China \\ ${ }^{2}$ Postdoctoral Research Station of Control Science and Engineering, Henan University of Science and Technology, Luoyang, \\ Henan 47100, China \\ ${ }^{3}$ Collaborative Innovation Center of Machinery Equipment Advanced Manufacturing of Henan Province, Luoyang, \\ Henan 471003, China \\ ${ }^{4}$ Luoyang Bearing Research Institute Co.,Ltd., Luoyang, Henan 471039, China \\ ${ }^{5}$ School of Mechanical Engineering, Northwestern Polytechnical University, Xi'an 710072, China
}

Correspondence should be addressed to Xiaoxu Pang; pxx8308@163.com

Received 20 May 2020; Revised 2 September 2020; Accepted 20 October 2020; Published 3 November 2020

Academic Editor: Francisco J. Montáns

Copyright (C) 2020 Xiaoxu Pang et al. This is an open access article distributed under the Creative Commons Attribution License, which permits unrestricted use, distribution, and reproduction in any medium, provided the original work is properly cited.

\begin{abstract}
Bearing ring residual stress test data obtained by $\mu$-360 s residual stress analyzer have the characteristics of unknown probability distribution and limited samples. For this problem, this study introduces the uncertainty and proposes a grey relation method to estimate the true value of the bearing ring residual stress. Based on poor information theory (incomplete and insufficient information for the characteristic presented in the subject investigated) and by fusing the membership function method, maximum membership method, rolling mean method, and bootstrap method, the true value sequence of the residual stress is obtained. On this basis, true value fusion is implemented again using the grey bootstrap method, and the estimated true value of the residual stress for the bearing ring was obtained. The results show that the residual stresses of bearing rings are fused by multiple methods, and the overall estimated true value of the residual stress of the bearing ring is $-578 \mathrm{MPa}$. Owing to different processing techniques, the true value of the residual stress varies in different parts of the ring as follows: $-918 \mathrm{MPa}$ on the large end surface of the ring, $-673 \mathrm{MPa}$ on the small end face of the ring, $-228 \mathrm{MPa}$ at the vertical test point on the outer surface of the ring, and $-231 \mathrm{MPa}$ at the parallel test point on the outer surface of the ring. The error between the estimated true value obtained using the grey relation method and that obtained through the fusion of multiple methods is generally $10 \%$ or less, thereby confirming the effectiveness of the grey relation method.
\end{abstract}

\section{Introduction}

Fatigue failure is one of the main failure forms for highspeed precision bearing. There are many factors that affect bearing fatigue failure, of which residual stress is one of the most important. As a result, the distribution of bearing residual stress has been increasingly researched in recent years $[1,2]$. Residual stresses are generated during the entire production cycle for bearings (including the turning, heat treatment, and grinding processes). These residual stresses include residual tensile stress and residual compressive stress [3]. During the bearing machining process, the residual stresses interact to form a residual compressive stress on the raceway. This residual compressive stress is beneficial for improving the fatigue life of the bearing [4].

During the processing of bearings, parameters including those for the turning process, heat treatment process, and grinding process will have an important impact on the formation of residual stress. It is difficult to model the formation of the stresses as the parameters and influences are complex. Therefore, many scholars have investigated the residual stress experimentally [5]. The experimental methods used can be divided into nondestructive and destructive test methods according to whether the parts are cut into samples in the experiments [6]. Nondestructive test methods include X-ray methods [7], ultrasonic methods [8], 
and neutron diffraction methods $[9,10]$; destructive testing methods include drilling methods [11] and delamination methods [12]. However, for both nondestructive and destructive test methods, because many factors will affect the residual stress value during the tests, the measured residual stress is relatively discrete, and the stress value has a large deviation $[13,14]$. As a result, the true value of the bearing residual stress difficultly be obtained accurately. Moreover, in the above literature, there are no reports focusing specifically on estimation of the true bearing residual stress. Therefore, it is necessary to estimate the true bearing residual stress according test data in order to provide theoretical guidance for investigating the influence of bearing residual stress on bearing life.

Owing to cost limitations, it is not feasible to obtain a large amount of bearing residual stress test data, and thus, it is not possible to determine the probability distribution of the data. Therefore, it is not possible to estimate the true value of the residual stress accurately using statistical methods. Xia et al. $[15,16]$ used the bearing friction torque as the data sequence and the bootstrap maximum entropy method as a bridge to integrate the Poisson process on the basis of the grey relation. As a result, the bearing performance stability and reliability were investigated, and the reliability model for the bearing quality was revised based on fuzzy mathematics theory; the calculation result of the revised model was verified to be higher than that of the original model, and the deviation was reduced by $8-21 \%$. Zhang et al. [17] combined the grey model-GM $(1,1)$, bootstrap method, and uncertainty theory to establish the grey bootstrap model for evaluating barometric pressure measurement results; the true estimation value and reliability of the barometric pressure were calculated. The results showed that the grey bootstrap model has better estimation accuracy than the bootstrap and GM $(1,1)$ methods. Pang et al. [18] used the bootstrap maximum entropy method to estimate the vibration reliability of a fan main shaft bearing and obtained an estimated true value of the bearing vibration. $\mathrm{Li}$ et al. $[19,20]$ analyzed reliability of long-life products under different measurement errors based on Wiener process. Palmieri and Ciuonzo [21, 22] proposed the maximum entropy solution (entropy priors) to solve the classification problems with lack of knowledge of the prior distribution, and the theory was verified on a small set of typical examples in comparison to uniform priors.

From the above analysis, it can be seen that domestic and foreign scholars have mainly carried out reliability and truth value assessments based on the poor information system, and used different methods to classify and merge data, and have achieved very beneficial results. The slight disadvantage is that the data fusion methods are used in a single method. Because different methods have different criteria, it will cause a certain deviation in the fusion result to a certain extent. This paper uses different methods to perform multiple fusions of the true value on the residual stress, and the result will be closer to the true value. In this study, the residual stress at different parts of the inner ring of a finished bearing is measured using an X-ray method. In addition, the residual stress is evaluated using the grey relation method, and a truth fusion method for the residual stress with the grey relation was proposed. At the same time, based on poor information theory, a combination of the membership function method, maximum membership method, rolling mean method, and bootstrap method are used to fuse the true value of the residual stress; the fusion true values obtained with these five methods comprise a true value fusion sequence. The fusion true value is obtained based on the true value fusion sequence through the grey bootstrap method, and then the estimated true value of the residual stress is obtained for different parts of the bearing inner ring. These results can provide theoretical guidance for improving the quality of bearing processing.

The rest of the paper is organized as follows: in Section 2, the principle of the proposed grey relation method and the five methods of membership function method, maximum membership method, rolling mean method, bootstrap method, and grey bootstrap method is firstly analyzed. In Section 3, the principle of the residual stress test is introduced and testing-related parameters are listed. In Section 4, the true values of the residual stress are obtained based on six methods, and through comparative analysis, the effectiveness of the proposed grey relation method is verified. In Section 5, the conclusion comes at the end of the paper.

\section{Truth Fusion Methods}

The true value is estimated in the poor information system. Due to the incomplete and insufficient information, it is necessary to use a variety of methods to study and obtain feature information from multiple aspects. Because different methods have different criteria, the obtained feature information is different, and these kinds of feature information are related to the true value. Therefore, according to the principle of information fusion, the feature information obtained by different methods is fused to form the set of feature information from different sides, and the feature information is merged again to estimate the true value more reasonably.

2.1. True Value Fusion Based on the Grey Relation. The residual stress data sequence for the bearing inner ring (the data are arranged from smallest to largest) can be obtained with a residual stress analyzer and represented by the following vector:

$$
X=\left[x_{1}(1), x_{2}(2), \ldots, x_{i}(k), \ldots, x_{n}(m),\right],
$$

where $x_{i}$ indicates the $i-$ th bearing ring, $i=1,2, \ldots, n$, and $x_{i}(k)$ is the $k$-th residual stress for a test location on the inner ring of the $i$ - th bearing.

2.1.1. Residual Stress Uncertainty. In order to make the residual stress test results more scientific, the uncertainty of the test results is needed to be assessed after completing the residual stress test. Uncertainty is the degree of uncertainty about the measured value due to measurement errors. The measurement uncertainty characterizes the reliability of the measurement results [23]. Tang et al. [24] proposed a 
weighted belief entropy based on Deng entropy in the frame of Dempster-Shafer evidence theory to quantify the uncertainty of uncertain information. The rationality and superiority of the new multisensor data fusion method are verified. The paper uses the following method to calculate uncertainty.

For analysis of the original data, when the confidence level is $95 \%$, the uncertainty expression is as follows:

$$
U_{i}(k)=K * U_{C}=K * \sqrt{\frac{\sum_{i=1}^{n} \sum_{k=1}^{m}\left(x_{i}(k)-\bar{x}_{i}(k)\right)^{2}}{m-1}},
$$

where $K$ is the inclusion factor, $K=2, U_{i}(k)$ is the synthetic uncertainty, and $\bar{x}_{i}(k)$ is the average residual stress.

2.1.2. Grey Relation Theory. Based on the grey system theory, the grey correlation degree is used to describe the relative relationship between data sequences. The grey relation is a certain inherent rule of the system. On the basis of sorting the data sequence, the attribute grey relation between two data sequences can be established. Assuming that the data sequence with the smallest uncertainty is the intrinsic sequence, defined as $Y_{1}(k)$, and the grey confidence level of the sequence is set to $100 \%$, the grey relationship can be established between the remaining $m-1$ sequences (defined as $\left.Y_{j}(k)\right)$ and the intrinsic sequence [25].

\subsubsection{Normalization. Let}

$$
\bar{y}_{h}=\frac{1}{k} \sum_{k=1}^{m-1} y_{h}(k), \quad h \in(1, j) .
$$

Thus,

$$
q_{h}(k)=\frac{v_{h}(k)-v_{h, \min }}{v_{h, \max }-v_{h, \min }},
$$

where

$$
\begin{aligned}
v_{h}(k) & =y_{n}(k)-\bar{y}_{n}, \\
v_{h, \min } & =\min v_{h}(k)=v_{h}(1), \\
v_{h, \max } & =\max v_{h}(k)=v_{h}(m-1) .
\end{aligned}
$$

The normalized sorting sequence for $Y_{h}$ can be expressed as

$$
Q_{h}=\left[q_{h}(k) \mid k=1,2, \ldots, m-1\right], \quad h \in(1, j) .
$$

For the normalized sequence, $Q_{h}$ :

$$
\begin{aligned}
q_{h}(k) & \in[0,1], \\
q_{h}(1) & =0, \\
q_{h}(m-1) & =1 .
\end{aligned}
$$

According to the principle of the least amount of information, all elements of the reference sequence, $Q_{O}$, are 0 . In other words,

$$
q_{O}(k)=q_{O}(1)=q_{O}(m-1)=0 .
$$

The grey correlation is defined as follows:

$$
\gamma_{O h}=\gamma\left(Q_{O}, Q_{h}\right)=\frac{1}{k} \sum_{k=1}^{\tau} \gamma\left(q_{O}(k), q_{h}(k)\right) .
$$

Taking the resolution coefficient as $\varepsilon \in(0,1]$, the expression for the grey correlation coefficient is

$$
\gamma\left(q_{O}(k), q_{h}(k)\right)=\frac{\varepsilon}{\Delta_{O h}(k)+\varepsilon} .
$$

Thus, the grey difference information can be expressed as follows:

$$
\Delta_{O h}(k)=\left|q_{h}(k)-q_{O}(k)\right| .
$$

The grey difference between the two data series, $Y_{1}$ and $Y_{j}$, is defined as

$$
d_{1 j}=\left|\gamma_{01}-\gamma_{0 j}\right|
$$

The similarity coefficient between data series $Y_{1}$ and $Y_{j}$ based on the grey correlation, which is referred to as the grey similarity coefficient, is given as follows:

$$
r_{1 j}=1-d_{1 j} \text {. }
$$

The grey similarity matrix is as follows:

$$
R=\left[\begin{array}{ll}
r_{11} & r_{1 j} \\
r_{j 1} & r_{j j}
\end{array}\right]=\left[\begin{array}{rr}
1 & r_{1 j} \\
r_{j 1} & 1
\end{array}\right]
$$

where $0 \leq r_{1 j} \leq 1$.

Given $Y_{1}$ and $Y_{j}$, there is always a unique real number $d_{\max }=d_{1 j \max }$ for $\varepsilon \in(0,1]$, such that $d_{1 j}<d_{\max }$, where $d_{\max }$ is the maximum grey difference, and the corresponding $\varepsilon$ is the optimal resolution coefficient based on the maximum grey difference.

The attribute weight based on the grey relation between data series $Y_{1}$ and $Y_{j}$ is defined as

$$
f_{1 j}= \begin{cases}1-\left(\frac{d_{\text {max }}}{\kappa}\right), & d_{\text {max }} \in[0, \kappa], \\ 0, & d_{\text {max }} \in[\kappa, 1],\end{cases}
$$

where $f_{1 j}$ is the attribute weight, $f_{1 j} \in[0,1]$, and $\kappa$ represents the $\kappa$ parameter, $\kappa \in[0,1]$.

2.1.4. Solving the Grey Confidence Level. According to the whitening principle and the symmetry principle of the grey system theory, if there is no reason to deny that $v$ is the true value, then under the given criteria, the default to represent the true value is $v$. In the two data sequences, $Y_{1}$ and $Y_{j}$, the parameter $v \in[0,1]$ is taken as the level. If there is a mapping of $f_{1 j} \geq v$, the data sequences $Y_{1}$ and $Y_{j}$ will have the same attributes. Here, taking $f_{1 j}=v=0.5$, it is considered that $Y_{1}$ and $Y_{j}$ have the same attributes.

Let $\kappa \in[0,0.5]$, then differentiating the $d_{\max }$ of equation (15) yields the following:

$$
d_{\max }=\left(1-f_{1 j}\right) \kappa .
$$


The grey confidence level (or grey theoretical probability) is thus as follows:

$$
P_{1 j}=1-(1-v) \kappa=(1-0.5 \kappa) \times 100 \% .
$$

The grey confidence level describes the degree of confidence degree for the same attributes in data series $Y_{1}$ and $Y_{j}$.

2.1.5. True Value Fusion. From the grey relation theory, if the relationship between the evaluation data series, $Y_{j}$, and the intrinsic data series, $Y_{1}$, is close, the value of the grey confidence level will be large, indicating that the grinding process is stable; in contrast, if the value of the grey confidence level is small, this means the grinding process is more unstable. The grey relation can be estimated based on the grey confidence level between the sequences and the data mean. According to the grey confidence level between the sequences and the mean value of the residual stress, the estimated true value is as follows:

$$
X_{01}=\frac{1}{m} \sum_{i=1}^{m} P_{1 i} \bar{x}_{i}(k) \text {. }
$$

2.2. True Value Fusion Based on the Membership Function Method. The membership function method is a quantitative fusion method.

Taking the data sequence in equation (1) as the original data sequence, the difference series, $d$, is defined as

$$
d=\left(d_{1}, d_{2}, \ldots, d_{i}, \ldots, d_{m-1}\right),
$$

where $d_{i}=x_{i+1}-x_{i}, i=1,2, \ldots, i, \ldots, m-1$.

Generally, the smaller the value of $d_{i}$, the denser the data values are. In other words, there is a relationship between $d_{i}$ and the distribution density of $x_{i}$. The linear membership function is defined as

$$
f_{i}=1-\frac{d_{i}-d_{\min }}{d_{\max }}, \quad i=1,2, \ldots, i, \ldots, m-1,
$$

where $d_{\min }=\min d_{i}$ and represents the minimum difference, and $d_{\max }=\max d_{i}$, representing the maximum difference.

The sequence of immediate values is defined as

$$
Z=\left(z_{1}, z_{2}, \ldots, z_{i}, \ldots, z_{m-1}\right)
$$

where $z_{i}=(1 / 2)\left(x_{i+1}+x_{i}\right), i=1,2, \ldots, m-1$.

Thus, based on the membership function method, the estimated true value is as follows:

$$
X_{02}=\frac{1}{\sum_{i=1}^{m-1} f_{i}} \sum_{i=1}^{m-1} f_{i} z_{i} \text {. }
$$

2.3. True Value Fusion Based on the Maximum Membership Method. Based on the membership function method, the maximum membership method is defined as

$$
f_{\text {max }}=\max f_{i}=1 \text {. }
$$

Therefore, taking the average of $x_{v+1}$ and $x_{v}$ corresponding to $f_{\max }$, the estimated true value, $X_{03}$, of the data series is as follows:

$$
\begin{array}{r}
X_{03}=\frac{1}{2}\left(x_{v+1}+x_{v}\right) \mid v, v+1 \longrightarrow f_{\max } \\
v \in i=(1,2, \ldots, m-1) .
\end{array}
$$

2.4. True Value Fusion Based on the Rolling Mean Method. Taking the data sequence in equation (1) as the original data sequence, the estimated true value is as follows:

$$
X_{04}=\frac{1}{n} \sum_{i=1}^{n} \xi_{i}
$$

where

$$
\xi_{j}=\frac{1}{m-j+1} \sum_{i=1}^{m-j+1} \sum_{k=i}^{i+j-1} \frac{x(k)}{j}, \quad j=1,2, \ldots, m .
$$

2.5. True Value Fusion Based on the Bootstrap Method. Taking the data sequence in equation (1) as the original data sequence, then based on the bootstrap principle, the bootstrap resampling sample, i.e., $X_{b}$, which contains $t$ data extracted $t$ times and one sample at a time, can be obtained through an equiprobable sampling with replacement from equation (1). As a result, B samples can be obtained [26], as follows:

$$
\begin{aligned}
X_{b} & =\left(x_{b}(1), x_{b}(2), \ldots, x_{b}(\tau), \ldots, x_{b}(t)\right), \\
b & =1,2, \ldots, B,
\end{aligned}
$$

where $X_{b}$ is the $b$ - th bootstrap sample; $x_{b}(\tau)$ is the $\tau-$ th data point of the $b$ - th bootstrap sample, $\tau=1,2, \ldots, t$; and $t$ is the number of the $b$ - th bootstrap sample. follows:

The average value of the bootstrap sample, $X_{b}$, is as

$$
X_{b}=\frac{1}{t} \sum_{k=1}^{t} x_{b}(\tau)
$$

The bootstrap sample with sample size $B$ is thus obtained:

$$
X_{\text {Bootstrap }}=\left(X_{1}, X_{2}, \ldots, X_{b}, \ldots, X_{B}\right) .
$$

By sorting $X_{\text {Bootstrap }}$ from small to large and dividing it into a group $Q$ (Table 1 ), the group median, $X_{m q}$, and discrete frequency of the bootstrap distribution, $F_{q}(q=1,2, \ldots, Q)$, can be obtained.

With frequency $F_{q}$ as the weight, the estimated true value based on the bootstrap method is as follows:

$$
X_{05}=\sum_{q=1}^{Q} F_{q} X_{\mathrm{mq}}
$$

2.6. True Fusion Based on the Grey Bootstrap Method. Let the data sequence for the estimated true value of the bearing residual stress obtained by $s$ methods be defined as follows: 
TABLE 1: Selection of the number of groups.

\begin{tabular}{lc}
\hline Sample size & Number of groups \\
\hline $50 \sim 60$ & $6 \sim 8$ \\
$60 \sim 100$ & $7 \sim 10$ \\
$100 \sim 200$ & $9 \sim 12$ \\
$200 \sim 500$ & $12 \sim 17$ \\
$>500$ & $<20$ \\
Computer simulation & $1.87(\mathrm{~B}-1)^{2.5}$ \\
\hline
\end{tabular}

$$
X_{s}=\left\{x_{01}, x_{02}, \ldots, x_{0 j}, \ldots, x_{0 s}\right\}, \quad j=1,2, \ldots, s,
$$

where $X_{0 j}$ represents the estimated true value obtained by method $j$, and $s$ is the number of methods, giving a total of $s$ data sequences.

Then, as in the bootstrap method, the bootstrap sample with sample size $B$ is obtained as follows:

$$
Y_{\text {Bootstrap }}=\left(Y_{1}, Y_{2}, \ldots, Y_{b}, \ldots, Y_{B}\right) \text {, }
$$

where $Y_{b}=\left\{y_{b}(e)\right\}, b=1,2, \ldots, B$.

Here, $y_{b}(e)$ is the $e-$ th bootstrap resampled data point of $Y_{b}$.

From the grey prediction of $\operatorname{GM}(1,1)$, the sequence vector generated by one accumulation is as follows [27]:

$$
X_{b}=\left\{x_{b}(e)\right\}=\left\{\sum_{j=1}^{e} y_{b}(j)\right\} \text {. }
$$

The grey generation model can be described by grey differential equations as follows:

$$
\frac{\mathrm{d} x_{b}(e)}{\mathrm{d} t}+c_{1} x_{b}(e)=c_{2}
$$

where $c_{1}, c_{2}$ are undetermined coefficients.

Using the incremental instead of differential method,

$$
\frac{\mathrm{d} x_{b}(e)}{\mathrm{d} t}=\frac{\Delta x_{b}(e)}{\Delta t}=x_{b}(e+1)-x_{b}(e)=y_{b}(e+1),
$$

where $\Delta e$ represents a unit time interval. follows:

The mean generation sequence vector is defined as

$$
\begin{array}{r}
Z_{b}=\left\{z_{b}(e)\right\}=\left\{0.5 x_{b}(e)+0.5 x_{b}(e-1)\right\}, \\
e=2,3, \ldots, t .
\end{array}
$$

Under the initial conditions, $x_{b}(1)=y_{b}(1)$. Thus, the least squares solution of the grey differential equation is

$$
\widehat{x}_{b}(j+1)=\left(y_{b}(1)-\frac{c_{2}}{c_{1}}\right) e^{-c_{1} j}+\left(\frac{c_{2}}{c_{1}}\right), j=e-1, e,
$$

where $\quad\left(c_{1}, c_{2}\right)^{T}=\left(D^{T} D\right)^{-1} D^{T}\left(Y_{b}\right)^{T}, D=\left(-Z_{b}, I\right)^{T}$, $I=(1,1, \ldots, 1)$.

From the cumulative production, the predicted value at time $w=e+1$ can be expressed as

$$
\hat{y}_{b}(w)=\hat{x}_{b}(w)-\hat{x}_{b}(w-1), \quad w=e+1 .
$$

At time $w$, the following vector can be formed from $B$ data:

$$
\widehat{X}_{w}=\left\{\widehat{y}_{b}(w)\right\} .
$$

As $B$ is very large, $\widehat{X}_{w}$ can be used to establish the value of the frequency function at time $e$ as follows:

$$
F_{w}=F_{w}\left(x_{t}\right),
$$

where $F_{w}$ is the grey bootstrap frequency function.

Thus, the estimated true value based on the grey bootstrap method is

$$
X_{0}=\sum_{q=1}^{Q} F_{\mathrm{wq}} x_{\mathrm{mq}}
$$

Using the maximum entropy and the Newton iteration method based on interval mapping, the probability density function for the total residual stress of the inner ring can be derived as follows [28]:

$$
f(x)=\exp \left[\sum_{k=0}^{M} \lambda_{k}(a x+b)^{k}\right]
$$

where $M$ is the order of the origin moment, $\lambda_{k}$ is the $(k)$-th Lagrange multiplier, and $a, b$ are mapping parameters.

The flowchart of the true value fusion based on different methods is shown in Figure 1. The advantages and disadvantages of different methods are shown in Table 2 .

\section{Principle of the Residual Stress Test}

The X-ray diffraction method determines the stress by measuring the distance between atoms based on the principle of Bragg's law [5]; the measured interatomic distance is closely related to the stress. This study uses the $\mu$-360 s residual stress analyzer produced by Japan PULSTEC to test the residual stress in different parts of the bearing inner ring. This method can measure the residual stress on the bearing surface without damaging the bearing, avoiding the disadvantage of causing stress relief by cutting the bearing. The test principle and stress calculation method are shown in Figure 2 and equation (43), respectively. The residual stress analyzer is shown in Figure 3. The parameters for the $\mu$-360 s residual stress analyzer and GCr15 material are listed in Tables 3 and 4, respectively.

$$
\begin{aligned}
\varepsilon_{\alpha} & =-\frac{1}{\tan \theta} \Delta \theta, \quad(0 \leq \alpha \leq 2 \pi), \\
\varepsilon_{\alpha 1} & =\frac{1}{2}\left[\left(\varepsilon_{\alpha}-\varepsilon_{\pi+\alpha}\right)+\left(\varepsilon_{-\alpha}-\varepsilon_{\pi-\alpha}\right)\right], \\
\sigma_{x} & =-\frac{E}{1+\nu} \cdot \frac{1}{\sin 2 \eta} \cdot \frac{1}{\sin 2 \psi_{0}} \cdot\left(\frac{\partial \varepsilon_{\alpha 1}}{\cos \alpha}\right),
\end{aligned}
$$

where $E$ is Young's modulus ( $\mathrm{MPa}), v$ is Poisson's ratio, $2 \theta$ is the diffraction angle $\left({ }^{\circ}\right), 2 \eta$ is the diffraction lattice angle $\left(^{\circ}\right)$, and $\varepsilon$ is the strain. 


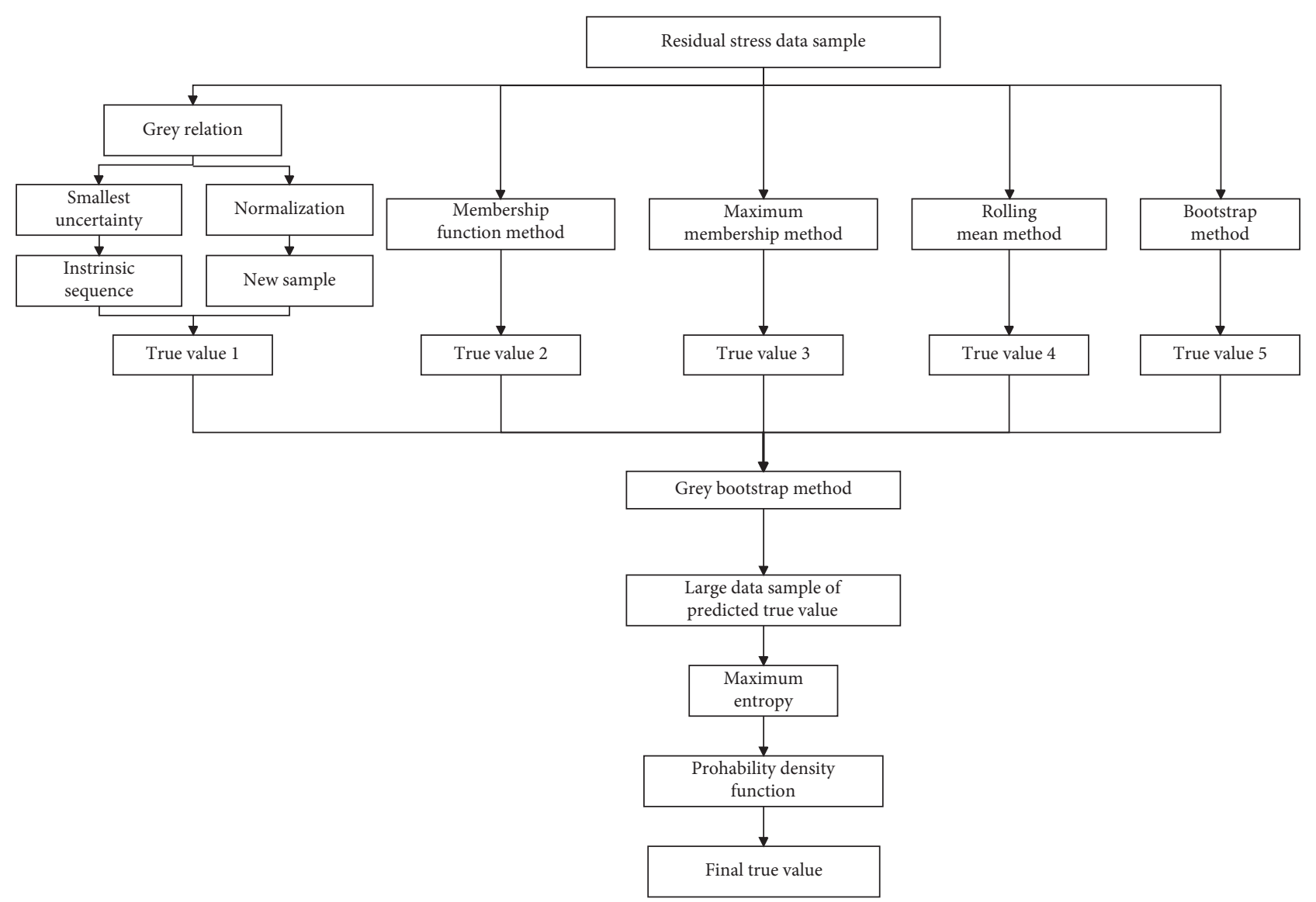

FIgURE 1: The flowchart of true value fusion.

TABLE 2: The advantages and disadvantages of different methods.

\begin{tabular}{|c|c|c|}
\hline Method & Advantages & Disadvantages \\
\hline Grey relation & Concern the closeness of data & Difficult to separate trend items \\
\hline $\begin{array}{l}\text { Membership function } \\
\text { method }\end{array}$ & Concern the distribution density among the data & $\begin{array}{l}\text { 1. Unknown membership function } \\
\text { 2. Difficultly calculate confidence level of the } \\
\text { evaluation result }\end{array}$ \\
\hline $\begin{array}{l}\text { Maximum membership } \\
\text { method }\end{array}$ & Concern the most densely distributed data & $\begin{array}{l}\text { 1. Too much depend on the original data } \\
\text { sequence distribution } \\
\text { 2. Difficultly calculate confidence level of the } \\
\text { evaluation result }\end{array}$ \\
\hline Rolling mean method & Concern the equal weight of the data & $\begin{array}{l}\text { 1. Difficult to separate trend items } \\
\text { 2. Difficultly calculate confidence level of the } \\
\text { evaluation result }\end{array}$ \\
\hline Bootstrap method & Concern the equal probability of data resampling & $\begin{array}{l}\text { 1.Little data } \\
\text { 2. Small confidence interval }\end{array}$ \\
\hline Grey bootstrap method & $\begin{array}{l}\text { Concern the development trend and probability weighting } \\
\text { of resampled data }\end{array}$ & Impossible multistep forecast \\
\hline
\end{tabular}

\section{Experimental Analysis}

4.1. Experimental Method. The $\mu-360$ s residual stress analyzer was used to carry out residual stress tests on four points of different parts of the five bearing inner rings after grinding processes. For each bearing, six evenly distributed points were tested to obtain the residual stress.

The residual stress was tested on four different parts of five bearing inner rings, and the bearing inner ring was ground (see Figure 4). Six residual stress values were obtained for each bearing, and the residual stress results are shown in Figure 5.

4.2. Residual Stress Evaluation Based on the Grey Relation. The residual stress values at different parts of the inner ring are normalized using equations (3) and (4), and the results are shown in Figure 6. 


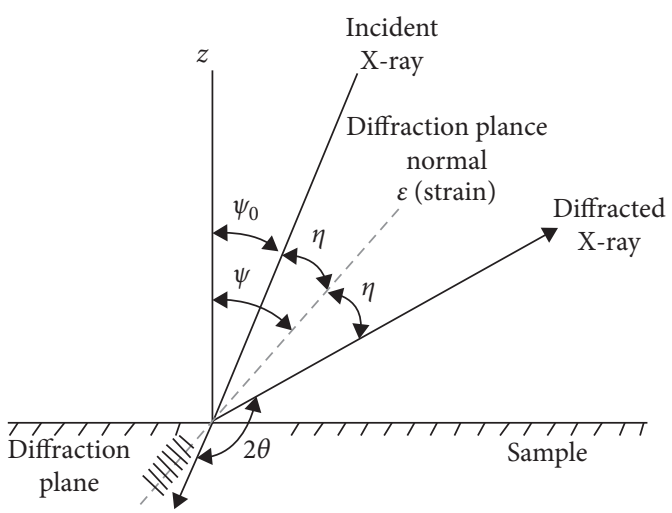

(a)

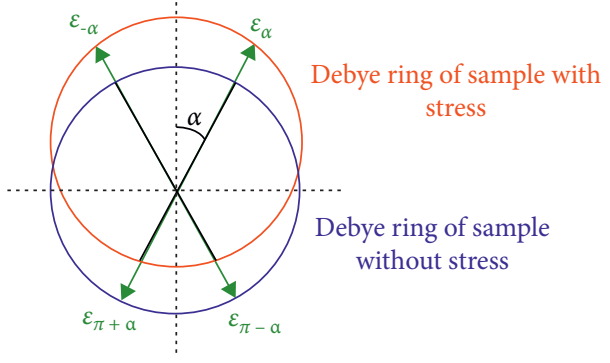

(b)

Figure 2: Principle of residual stress test.

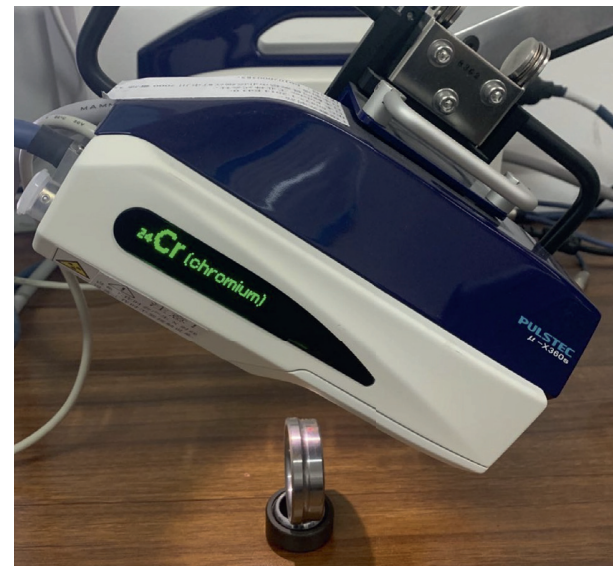

(a)

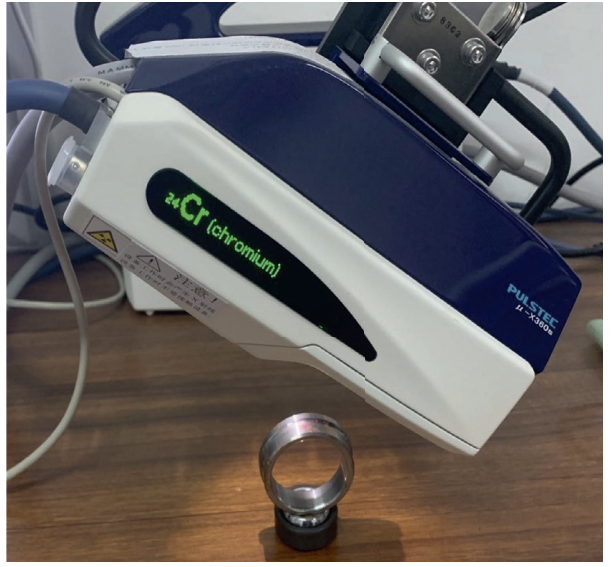

(b)

FIGURE 3: $\mu-360 \mathrm{~s}$ residual stress analyzer: (a) vertical test on the outer surface; (b) parallel test on the outer surface.

TABLE 3: Residual stress analyzer parameters.

\begin{tabular}{lccccc}
\hline X-ray tube & Bragg's angle $\left({ }^{\circ}\right)$ & Wavelength $(\mathrm{A})$ & Interval $(\mathrm{s})$ & Number of diffraction information & Voltage $(\mathrm{kV}) / \mathrm{current}(\mathrm{mA})$ \\
\hline Cr_K-alpha & 35 & 2.29093 & 15 & 500 & $30 / 1.5$ \\
\hline
\end{tabular}

TABLE 4: GCr15 material parameters.

\begin{tabular}{lcc}
\hline Density $\left(\mathrm{kg} / \mathrm{m}^{3}\right)$ & Elastic modulus $(\mathrm{GPa})$ & Poisson's ratio \\
\hline 7810 & 216 & 0.29 \\
\hline
\end{tabular}

The residual stress uncertainty can be obtained from equation (2). The minimum uncertainty data sequence is set as the intrinsic sequence, and the grey relation between the remaining residual stress data sequence and the intrinsic sequence is calculated. The results are summarized in Tables 5-8.

In Tables 5-8, the residual stress uncertainties of different parts of the bearing reveal that the intrinsic sequences of the large and small end surfaces of the inner ring are sample two data sequences, while the intrinsic sequences of the vertical and parallel tests of the outer surface of the inner ring are sample three data sequences. The remaining sequences are the comparison sequences. The confidence level of the comparison sequences for the intrinsic sequence varies widely, ranging from $77.8 \%$ to $96.85 \%$, which indicates that overall the processing technology is unstable during the inner ring processing. Compared with the outer surface of the inner ring (vertical and parallel tests), the grey confidence levels of the large and small end surfaces of the inner ring change more drastically and are mostly less than $90 \%$, while the grey confidence level of the outer surface of the inner ring is generally greater than $90 \%$. It can thus be seen that the processing technology for the large and small end surfaces of the inner ring is not as stable as the processing technology for the outer surface of the inner ring.

In Figure 6, it can be seen that after the residual stress value is normalized, the trends in the comparison sequence and intrinsic sequence are very different, which indicates that the processing technology for the bearing inner ring is unstable. The normalized values of the large and small end 


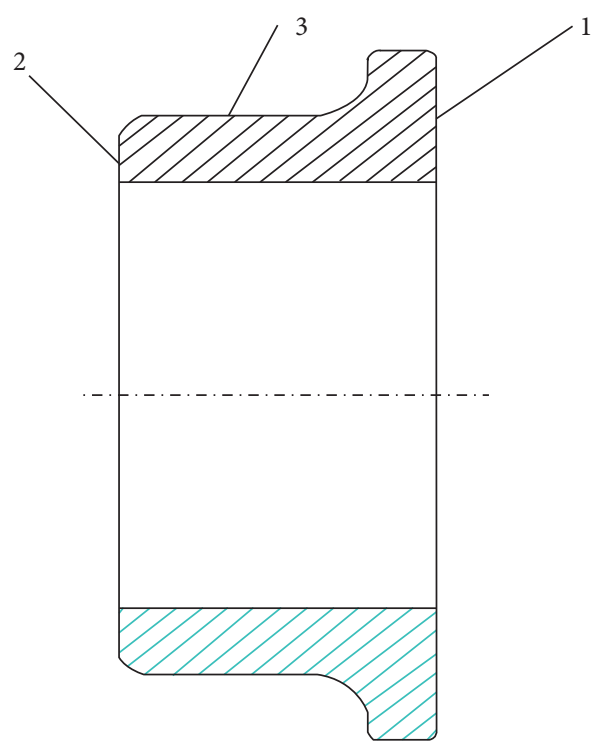

FIGURE 4: Schematic diagram of the different test parts of the inner ring. (1) Large end face; (2) small end face; (3) raceway (outer surface).

surfaces of the inner ring change more drastically than those of the outer surface, which indicates that the processing stability of the large and small end faces of the inner ring is not as good as the outer surface of the inner ring, consistent with the analysis based on the grey confidence level above.

From equation (18), the estimated true values of the residual stresses of different parts of the bearing inner ring can be obtained, as summarized in Table 9.

4.3. Evaluation of the True Residual Stress Based on the Membership Function Method. Taking the test data for different parts of the bearing inner ring as the original data, the true value of the residual stress can be obtained based on the membership function method, as shown Figure 7.

Subsequently, considering the estimated true values for different parts of each sample as the original data, the second fusion estimated true values of the residual stresses at different parts of the bearing inner rings can be obtained based on the membership function method again, as listed in Table 7.

4.4. Evaluation of the True Residual Stress Based on the Maximum Membership Method. Taking the test data for different parts of the bearing inner ring as the original data, the true value of the residual stress can be obtained based on the maximum membership method, as shown Figure 8.

Subsequently, considering the estimated true values for different parts of each sample as the original data, the second fusion estimated true values of the residual stresses at different parts of the bearing inner rings can be obtained based on the maximum membership method again, as listed in Table 9.

4.5. Evaluation of the True Residual Stress Based on the Rolling Mean Method. Taking the test data for different parts of the bearing inner ring as the original data, the true value of the residual stress can be obtained based on the rolling mean method, as shown Figure 9.

Subsequently, considering the estimated true values for different parts of each sample as the original data, the second fusion estimated true values of the residual stresses at different parts of the bearing inner rings can be obtained based on the rolling mean method again, as listed in Table 9.

4.6. Evaluation of the True Residual Stress Based on the Bootstrap Method. Considering the test data for different parts of the bearing inner ring as the original data, the true value of the residual stress can be obtained based on the bootstrap method, as shown in Figure 10.

Thereafter, the estimated true values for different parts of each sample are considered as the original data (e.g., the estimated true value of the residual stress of the large end surface). Applying the bootstrap method again with a bootstrap evaluation factor of $5, B=10000$, and a confidence level of $95 \%$ when the evaluation model is established, the bootstrap resampling sample of the residual stress on the large end face of the inner ring can be obtained, as shown in Figure 11. From equation (30), the second fusion estimated true value for the residual stress of the large end face of the bearing inner ring is $-858 \mathrm{MPa}$. Similarly, the estimated true values for the residual stresses of other parts of the inner ring are also obtained, as listed in Table 9.

In Figures 7-11, it can be seen that the estimated true value of the residual stress differs depending on the estimation method, and the results are different for the same batch of bearings. This is because when estimating the true value, the grey relation is concerned with the closeness of data, whereas the membership function method is concerned with the distribution density among the data, the maximum membership method is concerned with the most densely distributed data, the rolling mean method is 


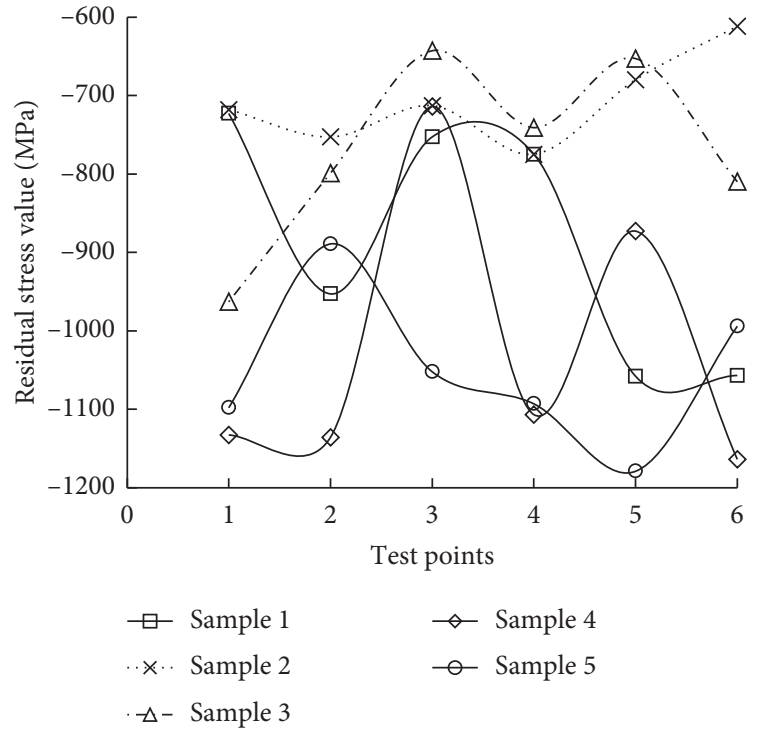

(a)

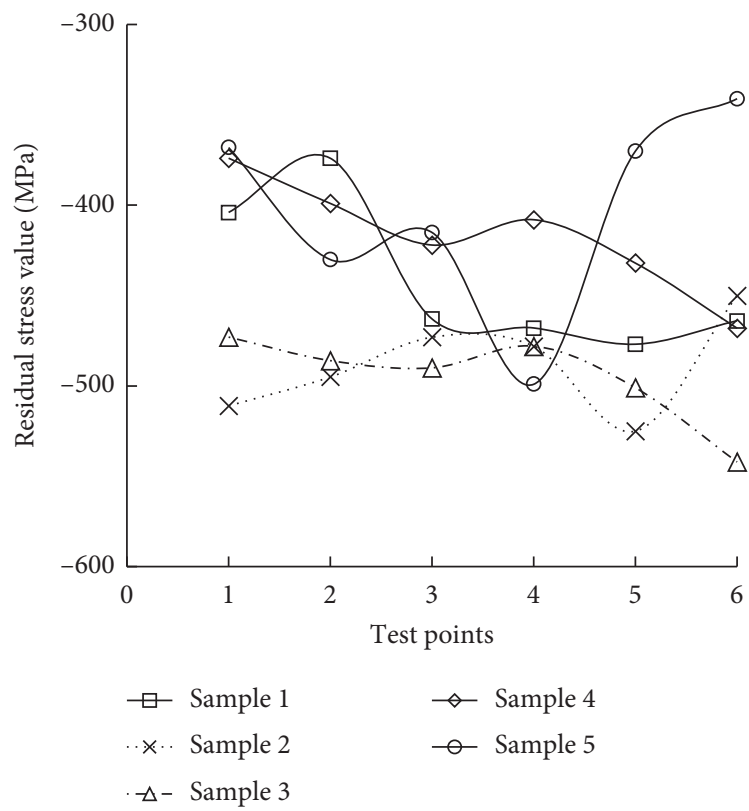

(c)

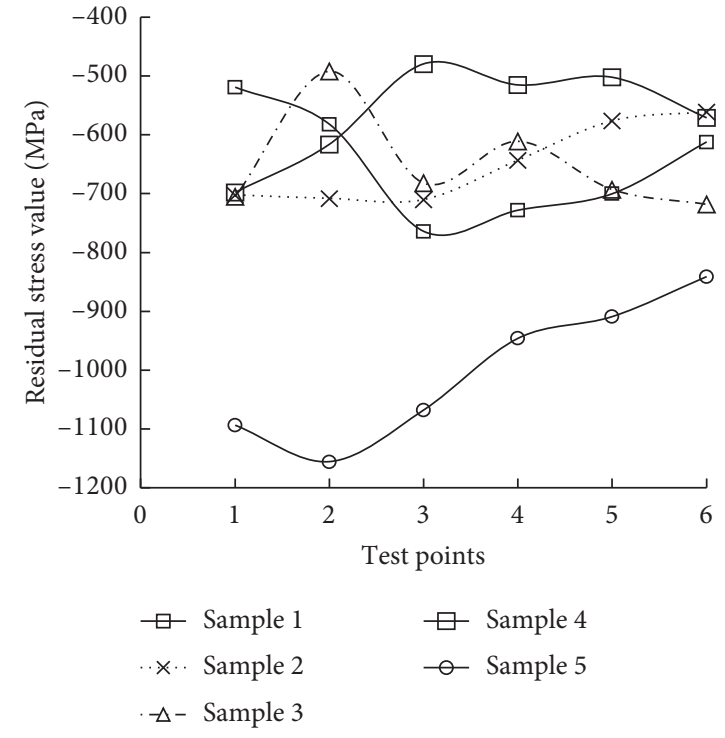

(b)

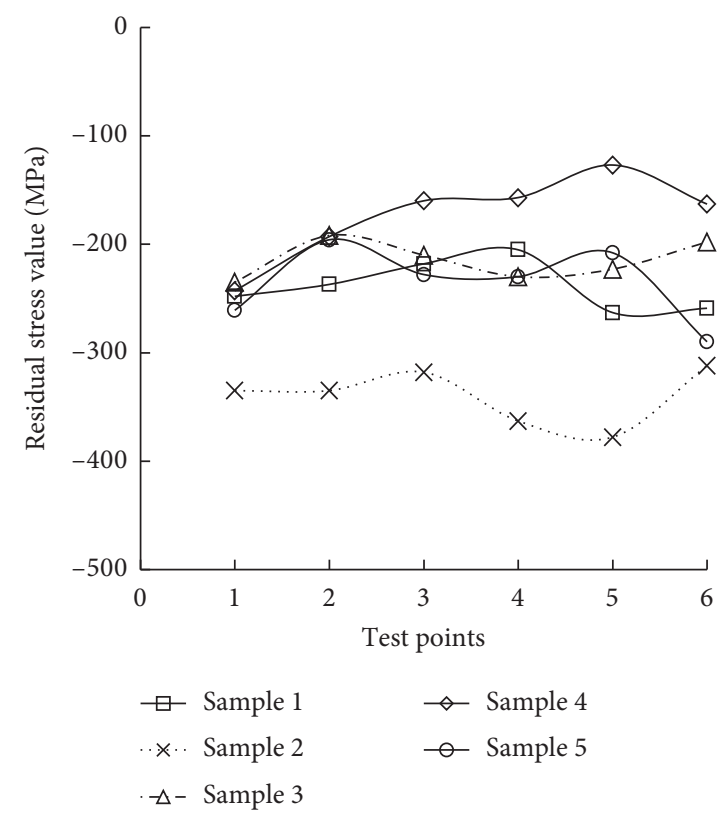

(d)

Figure 5: Residual stresses at different parts of the bearing inner ring. (a) Test point on the large end of the inner ring. (b) Test point on the small end of the inner ring. (c) Vertical test points on the outer surface of the inner ring. (d) Parallel test points on the outer surface of the inner ring.

concerned with the equal weight of the data, and the bootstrap method is concerned with the equal probability of data resampling.

In theory, the residual stress in the inner ring exists objectively, but the true value is unknown. In particular, the distribution of the residual stress on the inner ring remains unknown. Therefore, among these five methods, it is not possible to verify which estimation results are closest to the true value and most credible. These results can only show that these five methods describe five different side information sets regarding the inner ring. If more methods are used, additional side information could be obtained. By fusing multiple side information sets for the population, an overall estimated feature information set can be obtained. The more fusion information is included, the more accurate the estimated result will be.

4.7. Evaluation of the True Residual Stress Based on the Grey Bootstrap Method. In this study, the third fusion true values are obtained using the second fusion true values through the grey bootstrap method. The grey bootstrap method focuses on the development trend and probability weighting of resampled data. According to the grey bootstrap method 


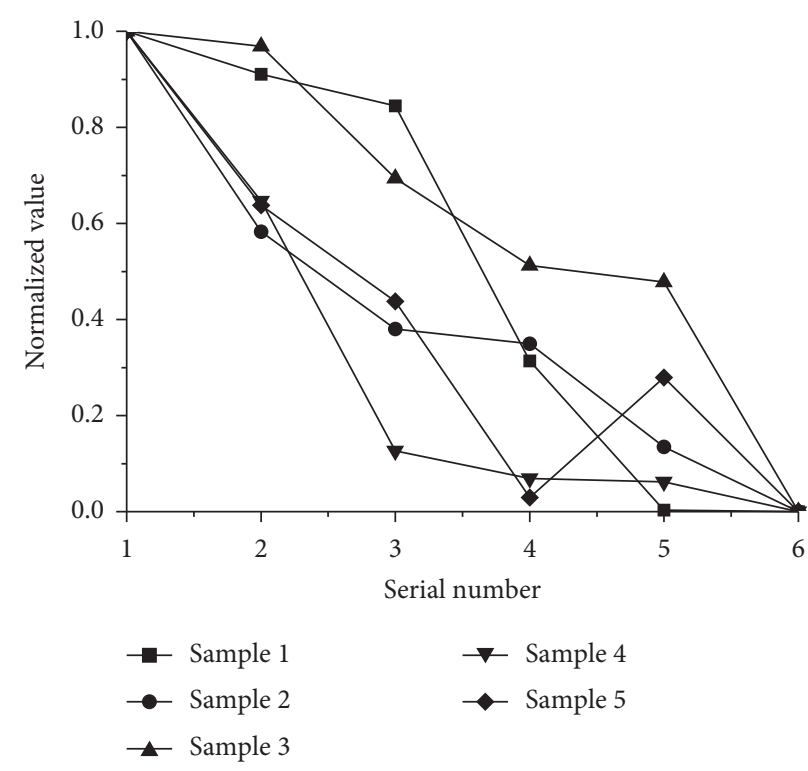

(a)

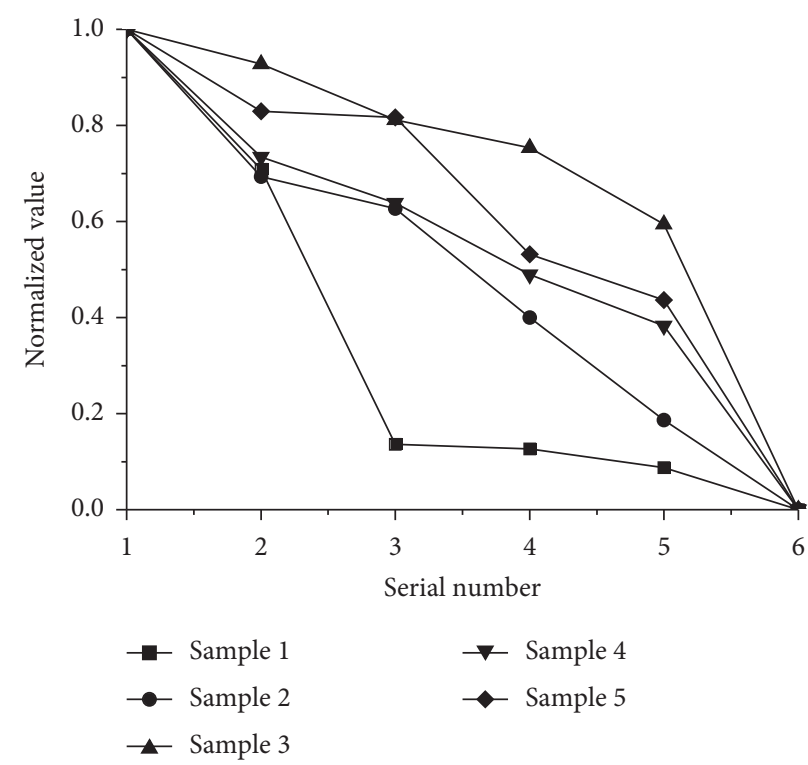

(c)

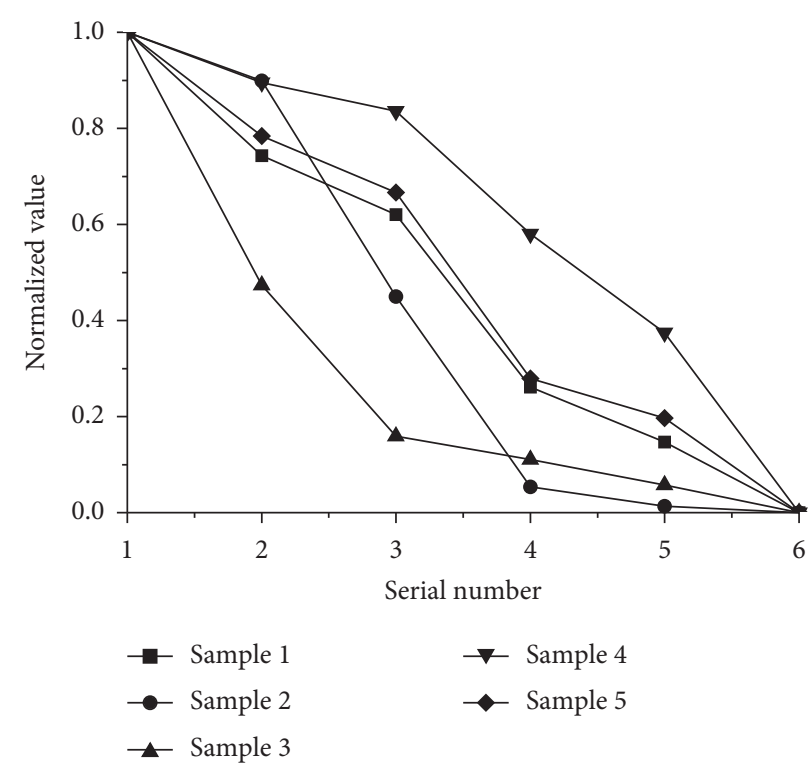

(b)

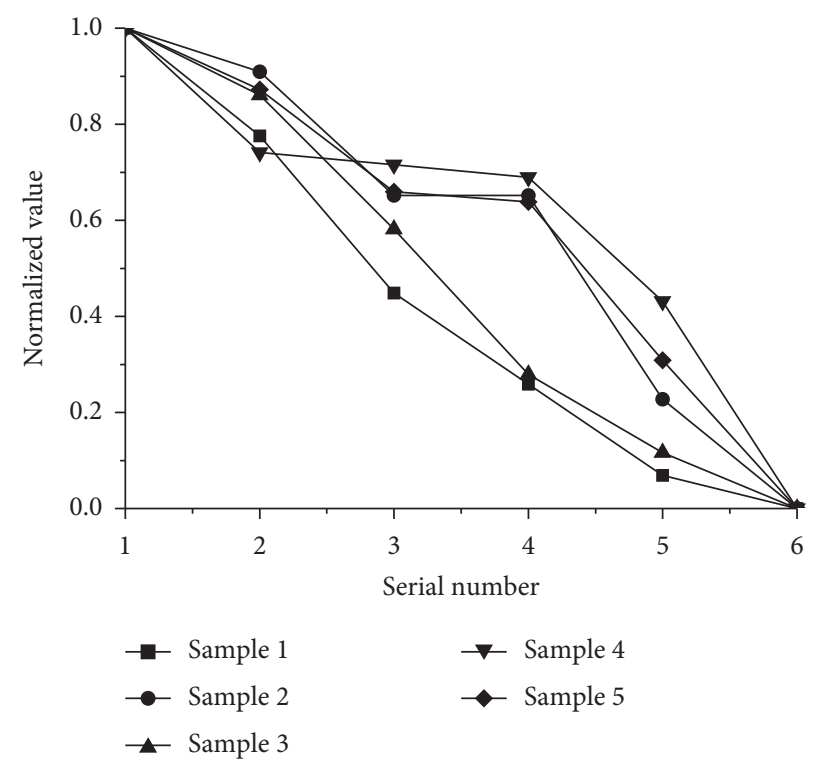

(d)

Figure 6: Normalized values of the residual stress at different test points on the bearing inner ring. (a) Large end of the inner ring. (b) Small end of the inner ring. (c) Outer surface of the inner ring (vertical test). (d) Outer surface of the inner ring (parallel test).

TABLE 5: Calculation results for the residual stress parameters of the large end faces of bearing inner rings.

\begin{tabular}{lccc}
\hline Sample number & Mean value $(\mathrm{MPa})$ & Uncertainty $(\mathrm{MPa})$ & Grey confidence level (\%) \\
\hline 1 & -887 & 309.7 & 90.025 \\
2 & -709 & 115.4 & 100 (intrinsic sequence) \\
3 & -768 & 237.2 & 89.175 \\
4 & -1021 & 368.5 & 86.5 \\
5 & -1051 & 199.7 & 96.85 \\
\hline
\end{tabular}

principle, the bootstrap evaluation factor is $5, B=10000$, and the confidence level is $95 \%$. Thus, the probability density function can be obtained, as shown in Figure 12, and the estimated true values are summarized in Table 10.

Comparing the grey relation method and grey bootstrap method results in Tables 9 and 10, respectively, reveals that the errors in the true residual stress values at different parts of the inner ring are $10.1 \%$ at the large end surface, $7.7 \%$ at the small end surface, $9.4 \%$ at the outer surface (the vertical test), and $1.2 \%$ at the outer surface (the parallel test). Therefore, the error in the true value between relative grey relation method and third fusion of the grey bootstrap 
TABLE 6: Calculation results for the residual stress parameters of the small end faces of bearing inner rings.

\begin{tabular}{lccc}
\hline Sample number & Mean value $(\mathrm{MPa})$ & Uncertainty $(\mathrm{MPa})$ & Grey confidence level (\%) \\
\hline 1 & -651 & 189.3 & 85.399 \\
2 & -650 & 136 & 100 (intrinsic sequence) \\
3 & -650 & 172.1 & 94.789 \\
4 & -564 & 165.4 & 78.975 \\
5 & -1002 & 243.7 & 83.8 \\
\hline
\end{tabular}

TABLE 7: Calculation results for the residual stress parameters of the outer surface of the bearing inner rings (the vertical test).

\begin{tabular}{lccc}
\hline Sample number & Mean value $(\mathrm{MPa})$ & Uncertainty $(\mathrm{MPa})$ & Grey confidence level (\%) \\
\hline 1 & -442 & 84.4 & 77.8 \\
2 & -489 & 54.5 & 90.67 \\
3 & -495 & 50 & 100 (intrinsic sequence) \\
4 & -417 & 63.9 & 94.465 \\
5 & -404 & 114 & 96.85 \\
\hline
\end{tabular}

TABLE 8: Calculation results for the residual stress parameters of the outer surface of the bearing inner rings (the parallel test).

\begin{tabular}{lccc}
\hline Sample number & Mean value $(\mathrm{MPa})$ & Uncertainty $(\mathrm{MPa})$ & Grey confidence level (\%) \\
\hline 1 & -238 & 46.1 & 96.685 \\
2 & -340 & 51.3 & 93.5 \\
3 & -215 & 35 & 100 (intrinsic sequence) \\
4 & -174 & 79.7 & 90.975 \\
5 & -236 & 69.4 & 92.38 \\
\hline
\end{tabular}

TABLE 9: Second fusion estimation of the true value for the residual stress at different parts of the bearing inner ring under different methods.

\begin{tabular}{lcccc}
\hline & Large end (MPa) & Small end (MPa) & $\begin{array}{c}\text { Outer surface } \\
\text { (vertical test) }(\mathrm{MPa})\end{array}$ & $\begin{array}{c}\text { Outer surface } \\
(\mathrm{parallel} \text { test) }(\mathrm{MPa})\end{array}$ \\
\hline Grey relation method & -819 & -621 & -413 & -228 \\
Membership function method & -895 & -654 & -461 & -220 \\
Maximum membership method & -1077 & -712 & -476 & -231 \\
Bootstrap method & -858 & -670 & -457 & -239 \\
Rolling mean method & -892 & -693 & -449 & -238 \\
\hline
\end{tabular}

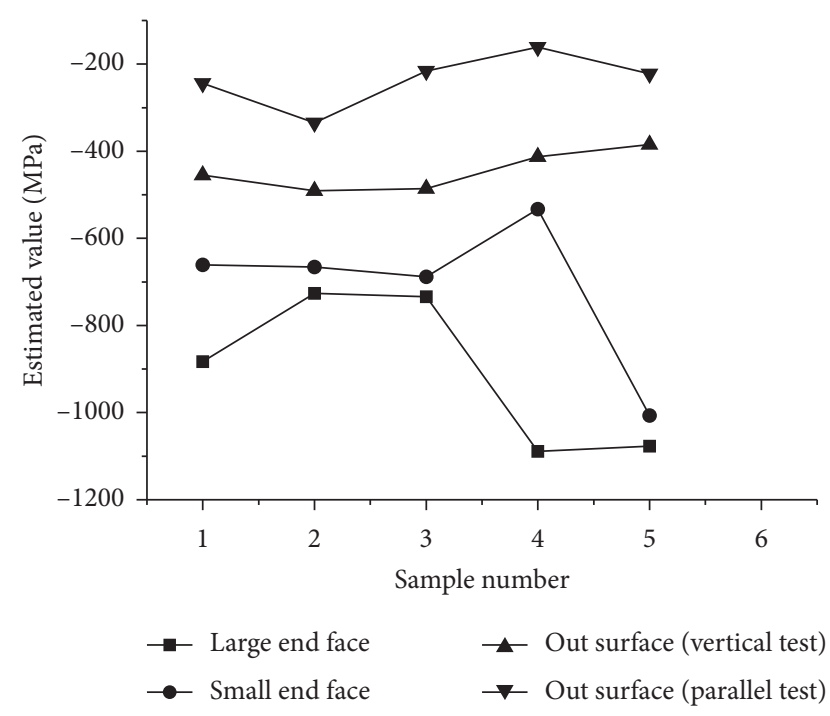

FIGURE 7: Estimated true values of the residual stresses at different parts of the inner ring based on the membership function method.

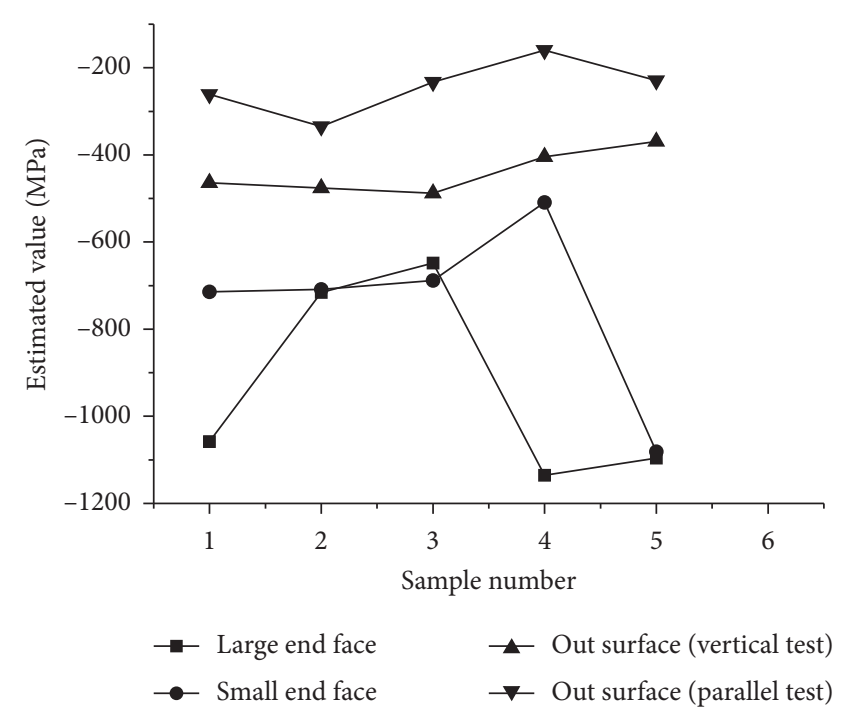

FIgURE 8: Estimated true values of the residual stress of different parts of the inner ring based on the maximum membership method. 


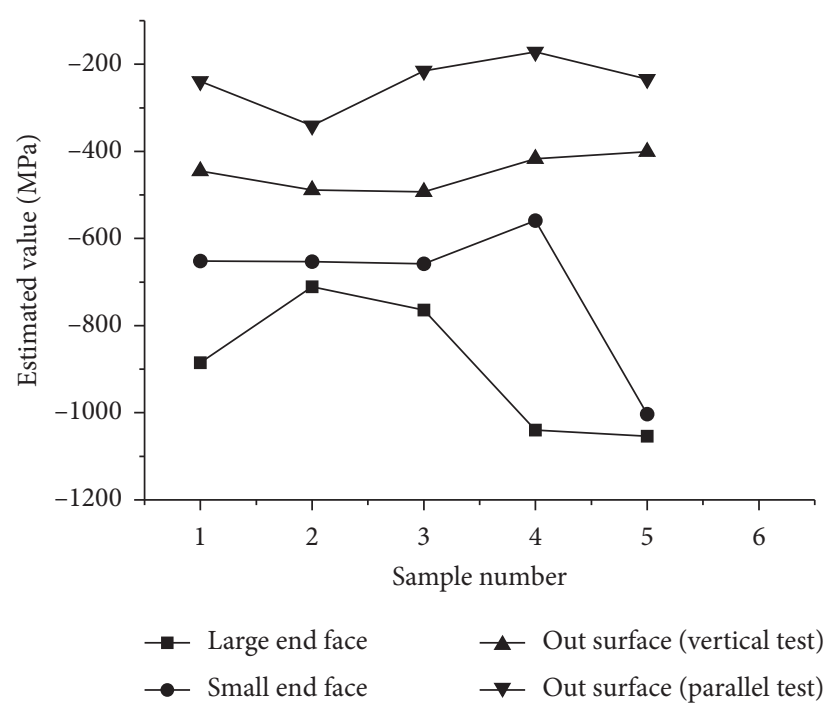

FIgURE 9: Estimated true values of the residual stress of different part of the inner ring based on the rolling mean method.

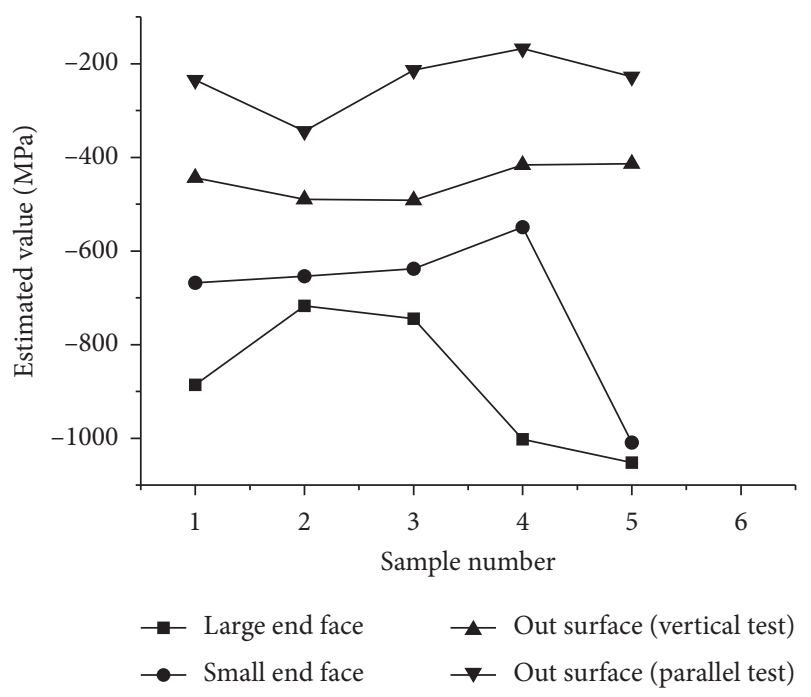

Figure 10: Estimated true values for the residual stresses at different parts of the inner ring based on the bootstrap method.

method is less than $10 \%$, which further verifies that the grey relation method can estimate the true value of the residual stress. Owing to an outlier of $-1077 \mathrm{MPa}$ in the estimated true values, the error in the true residual stress value for the large end face is greater than $10 \%$.

Table 10 lists the estimated true values of the residual stress at different parts of the inner ring. From Table 10, it is evident that the true residual stress values at different parts of the inner ring vary significantly. The residual stress at the large end face is the highest, whereas that at the outer surface (parallel test) is the lowest. These residual stress values are related to the processing technologies used for different parts of the inner ring.

The large end face and the small end face have similar left-skewed residual stress distributions, as shown in Figures 12(a) and 12(b); the residual stress distributions of

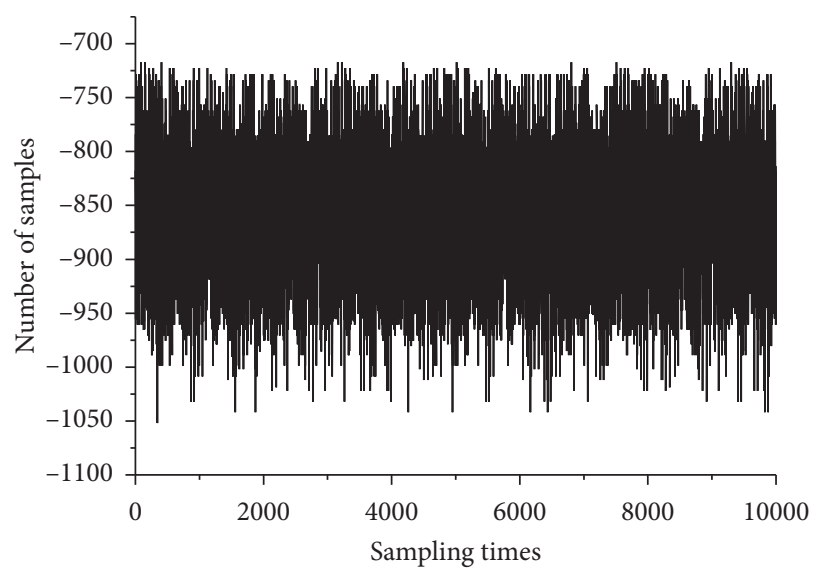

FIGURE 11: Bootstrap resampling sample of the residual stress on the large end face.

the outer surface (vertical and parallel tests) are rightskewed. Because the processing technology differs for different parts of the inner ring, the skews of the probability density function distributions are also different. A small peak appears in the probability density function for the large end surface because an abnormal data point of $-1077 \mathrm{MPa}$ appears when the true value of the residual stress is estimated based on the maximum membership method. The estimated true residual stress value at different parts of the bearing is a type of individual feature information. To obtain the true residual stress value of the entire inner ring, further data fusion is required.

The estimated true value in Table 10 is used to form the new intrinsic fusion sequence, and the new fusion sequence is processed again using the grey bootstrap method. The resulting overall estimated true value of the residual stress of the inner ring for the batch of bearings is $-578 \mathrm{MPa}$, and the probability density function is shown in Figure 13. From Figure 13, it can be seen that the true value 


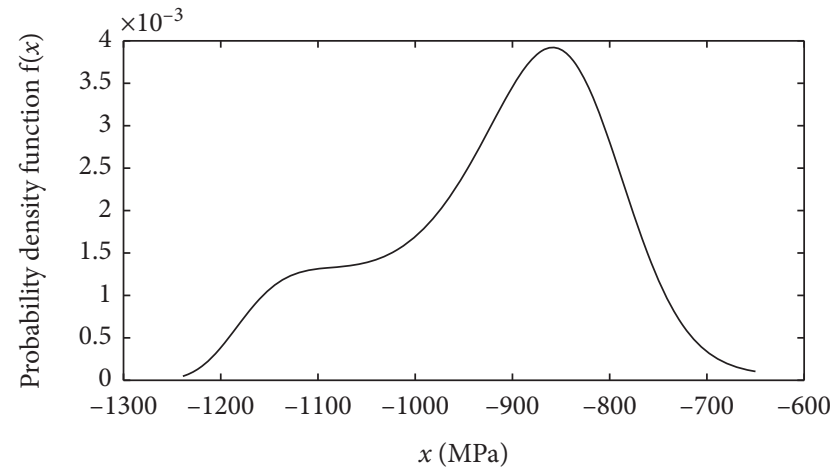

(a)

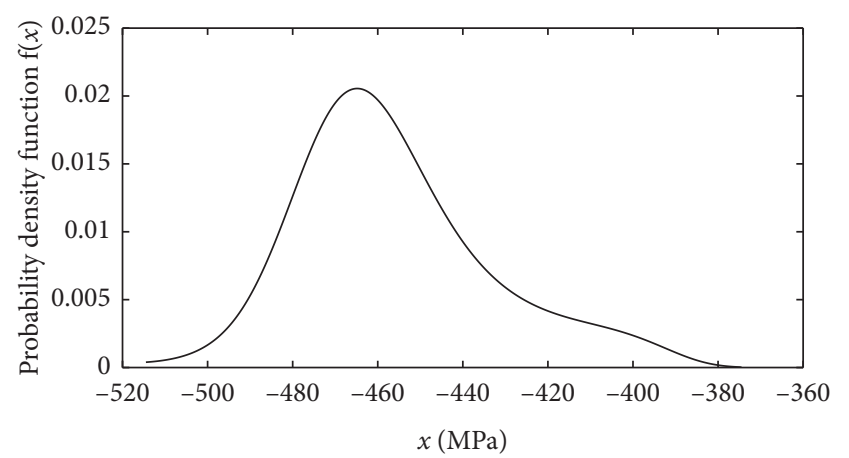

(c)

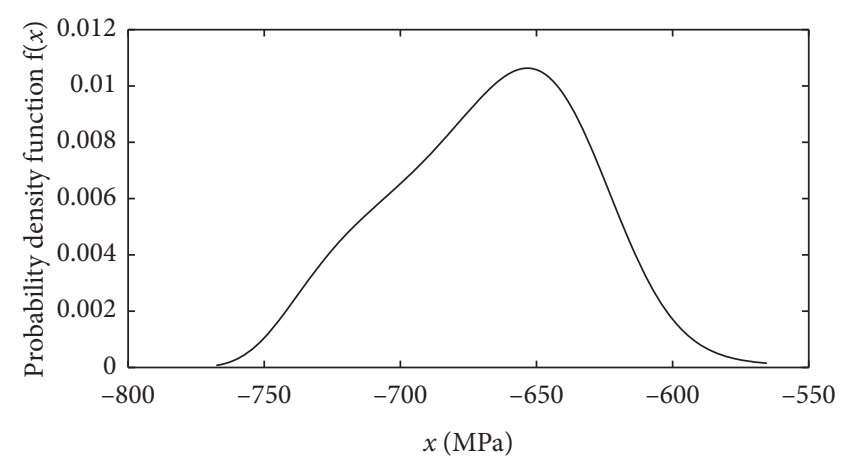

(b)

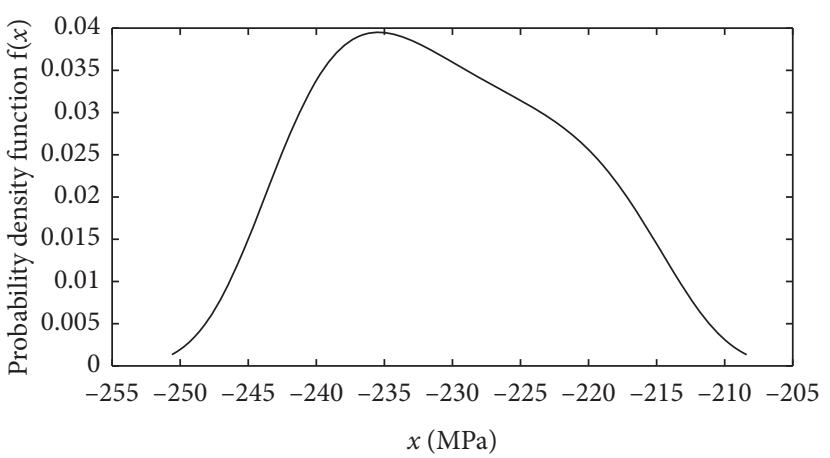

(d)

FIGURE 12: Probability density functions for the residual stress at different parts of the bearing inner ring. (a) Probability density function for the residual stress of the large end face. (b) Probability density function for the residual stress of the small end face. (c) Probability density function for the residual stress of the outer surface (the vertical test). (d) Probability density function for the residual stress of the outer surface (the parallel test).

TABLE 10: Third fusion estimated true values of the residual stress at different parts of the bearing inner ring based on the grey bootstrap method.

\begin{tabular}{lcccc}
\hline & $\begin{array}{c}\text { Large end } \\
(\mathrm{MPa})\end{array}$ & $\begin{array}{c}\text { Small end } \\
(\mathrm{MPa})\end{array}$ & $\begin{array}{c}\text { Outer surface } \\
\text { (vertical test) }(\mathrm{MPa})\end{array}$ & $\begin{array}{c}\text { Outer surface } \\
(\mathrm{parallel} \mathrm{test)}(\mathrm{MPa})\end{array}$ \\
\hline Grey bootstrap method & -918 & -673 & -456 & -231 \\
\hline
\end{tabular}

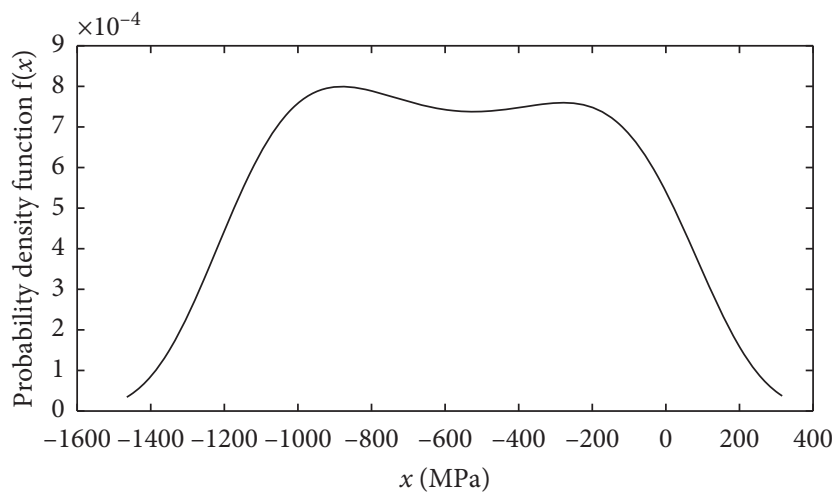

FIGURE 13: Probability density function for the overall true residual stress value. 
distribution of the inner ring is not a normal distribution, but rather a bimodal distribution, which is also caused by the different processing techniques used for different parts of the inner ring.

\section{Conclusions}

This study employs a residual stress analyzer to test the residual stresses of different parts of a bearing inner ring after grinding, and the estimated true values of the residual stress for the bearing inner ring are obtained through various methods. The following conclusions are drawn:

(1) Introducing uncertainty, a method for evaluating the bearing grinding stability and estimating the true value of the residual stress based on the grey relation theory is proposed, and the estimated true values of the residual stress for different parts of a bearing inner ring are obtained.

(2) Using a fusion of the grey relation method, membership function method, maximum membership method, rolling mean method, and bootstrap method, the true values of the residual stress in different parts of the inner ring are estimated. Thereafter, on the basis of obtaining the second fusion true values, the true values are estimated again based on the grey bootstrap method, allowing the final true values of the residual stress to be estimated in different parts of the inner ring. As a result of the different processing technologies used for different parts of the inner ring, the true residual stress values of different parts vary greatly. The probability density functions of the residual stress are also different: the large end face and small end face are right-skewed, while the outer surface (vertical and parallel tests) of the inner ring is left-skewed.

(3) Compared with the final true values based on the grey bootstrap method, the true residual stress values based on the grey relation give an error of generally less than $10 \%$, which further verifies the correctness of the grey relation method.

(4) Using fusion true values based on multiple methods, the overall true value of the bearing inner ring estimated based on the grey bootstrap method is $-578 \mathrm{MPa}$, and the probability density function is bimodal.

\section{Data Availability}

The residual stress value data used to support the findings of this study are included within the supplementary material.

\section{Conflicts of Interest}

The authors declare that they have no conflicts of interest.

\section{Acknowledgments}

The research was supported by the National Natural Science Foundation of China (grant no. 51805151) and Key
Specialized Research and Development Breakthrough in Henan Province (grant no. 202102210083). The authors gratefully acknowledged the helpful discussions with the research group and colleagues in the School of Mechanical and Electrical Engineering at Henan University of Science and Technology, and the authors would like to thank Editage (http://www.editage.cn) for English language editing.

\section{Supplementary Materials}

The supplementary material is the original data of the residual stress on four points of different parts of the five bearing ring. For each bearing ring, six distributed points were tested to obtain the residual stress. (Supplementary Materials)

\section{References}

[1] D. Wang, P. Ge, W. Bi et al., "Experimental study on residual stress distribution in surface layer of rolling bearing inner ring raceway," Journal of Huazhong University of Science and Technology (Nature Science Edition), vol. 43, no. 3, pp. 12-16, 2015.

[2] R. Yang, P. Chen, J. Wang et al., "Effects of residual stress and its characterization parameters on rolling contact performance of materials," Journal of Sichuan University (Engineering Science Edition), vol. 48, no. 4, pp. 211-216, 2016.

[3] Y. Choi, "A study on the effects of machining-induced residual stress on rolling contact fatigue," International Journal of Fatigue, vol. 31, no. 10, pp. 1517-1523, 2009.

[4] F. Pape, T. Neubauer, O. Maiß, B. Denkena, and G. Poll, "Influence of residual stresses introduced by manufacturing processes on bearing endurance time," Tribology Letters, vol. 65, no. 2, pp. 65-707, 2017.

[5] Y. He, J. Zhou, Q. Zhou et al., "Technological experiment and regression analysis of surface residual stress in ultrasonic grinding," Journal of Southwest Jiaotong University, vol. 52, no. 3, pp. 612-617, 2017.

[6] F. Wang, K. Mao, and B. Li, "Prediction of residual stress fields from surface stress measurements," International Journal of Mechanical Sciences, vol. 140, pp. 68-82, 2018.

[7] J. Zhou, J. Ren, and C. Ju, "Prediction of residual stress for machining aviation engine blade based on RBF neural network," Computer Integrated Manufacturing Systems, vol. 24, no. 2, pp. 361-370, 2018.

[8] M. Li, H. Sun, G. Gou, and J. Yu, "Analysis of residual-stress test errors via ultrasonic method for aluminium alloy used in high-speed trains," Journal of Southwest Jiaotong University, vol. 53, no. 6, pp. 1295-1300, 2018.

[9] D. Chang, J. Zhang, Y. Lian et al., "Research progress on residual stress in laser additive manufacturing," Surface Technology, vol. 48, no. 1, pp. 200-207, 2019.

[10] V. Bedekar, R. Voothaluru, J. Bunn, and R. S. Hyde, "Measurement and prediction of through-section residual stresses in the manufacturing sequence of bearing components," CIRP Annals, vol. 68, no. 1, pp. 57-60, 2019.

[11] S. Gan, Y. Han, and F. Chen, "Analysis of error factors in measuring welding residual stress by drilling," Transactions of the China Welding Institution, vol. 39, no. 10, pp. 48-53, 2018.

[12] X. Liu, C. Zhao, L. Fan et al., "Analysis of residual stress in bearing rings of reinforced grinding," Modular Machine Tool \& Automatic Manufacturing Technique, no. 9, pp. 5-8, 2017. 
[13] F. Wang, K. Mao, S. Wu, B. Li, and G. Xiao, "Bivariate Fourier-series-based prediction of surface residual stress fields using stresses of partial points," Mathematics and Mechanics of Solids, vol. 24, no. 4, pp. 979-995, 2019.

[14] K. Liao and Y. Wu, "Uncertainty of stress determination by LRM in aluminum alloy thick plate," Materials Science and Technology, vol. 22, no. 4, pp. 86-90, 2014.

[15] X. Xia, Z. Chang, Y. Liang et al., "Stability and reliability analysis of rolling bearing performance," Chinese Journal of Scientific Instrument, vol. 38, no. 6, pp. 1421-1431, 2017.

[16] X. Xia, B. Liu, Y. Li et al., "Research on quality achieving reliability of bearing based on fuzzy weight," Journal of Aerospace Power, vol. 33, no. 12, pp. 3013-3021, 2018.

[17] L. Zhang, Y. Song, X. Wang et al., "Evaluation method for dynamic measurement result of atmospheric pressure based on grey bootstrap model," Chinese Journal of Scientific Instrument, vol. 38, no. 7, pp. 1645-1652, 2017.

[18] X. Pang, M. Qiu, Y. Liang et al., "Vibration reliability evaluation of main fan spindle bearings," Shock and Vibration, vol. 2019, Article ID 8182530, 12 pages, 2019.

[19] J. Li, Z. Wang, C. Liu, and M. Qiu, "Stochastic accelerated degradation model involving multiple accelerating variables by considering measurement error," Journal of Mechanical Science and Technology, vol. 33, no. 11, pp. 5425-5435, 2019.

[20] J. Li, Z. Wang, Y. Zhang, C. Liu, and H. Fu, "A nonlinear Wiener process degradation model with autoregressive errors," Reliability Engineering \& System Safety, vol. 173, pp. 48-57, 2018.

[21] F. Palmieri and D. Ciuonzo, "Objective priors from maximum entropy in data classification," Information Fusion, vol. 14, no. 2, pp. 186-198, 2013.

[22] F. Palmieri and D. Ciuonzo, "Data fusion with entropic priors," in Proceedings of the 20th Italian Workshop on Neural Networks, pp. 107-114, Salerno, Italy, May 2010.

[23] B. Qiang, Y. Li, Y. Gu et al., "Experimental study on welding residual stress spatial distribution in thick plate for main member of steel truss bridge," Journal of the China Railway Society, vol. 41, no. 3, pp. 128-133, 2019.

[24] Y. Tang, D. Zhou, S. Xu et al., "A weighted belief entropybased uncertainty measure for multi-sensor data fusion," Sensors, vol. 17, no. 4, p. 928, 2017.

[25] X. Xia, Y. Qin, and M. Qiu, "Evaluation for stability of manufacturing process based on grey relation," Journal of Aerospace Power, vol. 30, no. 3, pp. 762-768, 2015.

[26] X. Xia, "Reliability analysis of zero-failure data with poor information," Quality and Reliability Engineering International, vol. 28, no. 8, pp. 981-990, 2012.

[27] X. Xia, Y. Meng, B. Shi et al., "Bootstrap forecasting method of uncertainty for rolling bearing vibration performance based on $\operatorname{GM}(1,1)$," The Journal of Grey System, vol. 7, no. 2, pp. 78-92, 2015.

[28] Y. Liang, X. Xia, and C. Zhen, "Evaluation of dynamic uncertainty of rolling bearing vibration performance," Mathematical Problems in Engineering, vol. 2019, p. 17, Article ID 2896046, 2019. 